MN2006-17028

\title{
Multifunctional Characteristics of Carbon Nanotube (CNT) Yarn Composites
}

\author{
Corey D. Hernandez \\ Swales Aerospace \\ Hampton, VA 23681
}

\author{
Mei Zhang, Shaoli Fang, and \\ Ray H. Baughman \\ University of Texas at Dallas \\ Richardson, TX
}

\author{
Thomas S. Gates and \\ Seun K. Kahng* \\ NASA Langley Research Center \\ Hampton, VA 23681 \\ *seun.k.kahng@nasa.gov
}

\begin{abstract}
By forming composite structures with Carbon Nanotube (CNT) yarns we achieve materials capable of measuring strain and composite structures with increased mechanical strength. The CNT yarns used are of the 2ply and 4-ply variety with the yarns having diameters of about 15-30 $\mu \mathrm{m}$. The strain sensing characteristics of the yarns are investigated on test beams with the yarns arranged in a bridge configuration. Additionally, the strain sensing properties are also investigated on yarns embedded on the surface of a flexible membrane. Initial mechanical strength tests also show an increase in the modulus of elasticity of the composite materials while incurring a weight penalty of less than one-percent. Also presented are initial temperature characterizations of the yarns.
\end{abstract}

With the expansion of the performance envelope of aerospace vehicles and systems, designers are more often turning to the selective use of light-weight, multifunctional materials. This trend has forced many organizations to place an increased emphasis on the ability to find material candidates that meet the strict aerospace vehicle requirements [1]. Candidate systems suitable for multifunctionality may include the health monitoring systems of inflatable structures, manned space habitats, manned space suits and other aerospace vehicles and space structures.

The use of CNT yarns in part is motivated by the high strength and mechanical properties found in the individual carbon nanotubes that comprise yarn bundles [2]. While major advances have been made in yarn spinning, no one has yet been able to realize the inherent mechanical, electrical, or thermal properties of the individual carbon nanotubes in such nanotube assemblies [3-9].

The spun CNT yarns used in this work are 2- and 4-ply and have lengths of over 10-meters and diameters of about 15 to $30 \mu \mathrm{m}$. These yarns have tensile strengths of about $460 \mathrm{MPa}$ and effective yarn tensile moduli greater than $20 \mathrm{GPa}$. Electrical conductivity is found to be $300 \mathrm{~S} / \mathrm{cm}$ at room temperature and have a negative temperature dependence of resistance of $0.1 \%$ per degree $\mathrm{C}$. They have also been shown to retain their strength and flexibility after heating in air to $+460^{\circ} \mathrm{C}$ for an hour and when immersed in liquid nitrogen at $-196^{\circ} \mathrm{C}$ [8]. The yarn fabrication process is explained in detail in reference 8 and a scanning electron microscope (SEM) micrograph of a 4-ply yarn bundle is shown in Figure 1.

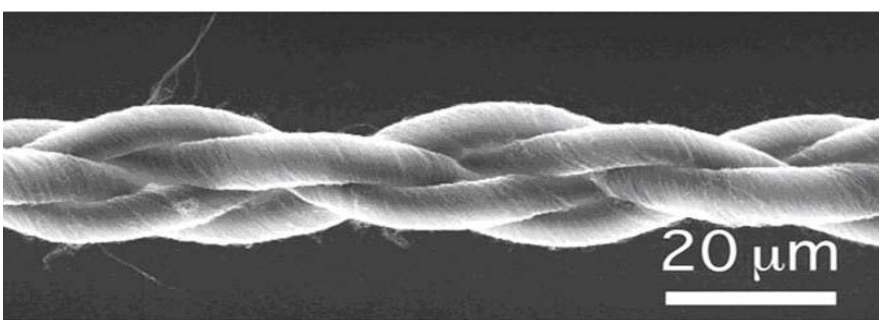

Figure 1. SEM image of a 4-ply carbon nanotube yarn [8].

The present objective is to report on results of the initial steps taken at both the NanoTech Institute at the University of Texas at Dallas [10-13] and NASA Langley Research Center to incorporate CNT yarns into composite structures that provide multifunctionality through mechanical strength enhancement and strain sensing. 
Three primary experiments are reported towards the development of multifunctional structures utilizing CNT yarns. The experiments presented are: 1) strain sensitivity of the CNT yarns using a cantilever test beam, 2) tensile strength tests of thin composite samples reinforced with the CNT yarns, and 3) average strain measurement along CNT yarns embedded in a thin membrane.

In all three presented experiments researchers utilized CNT yarns embedded in polyurethane and silicon rubber. To further investigate the degree of bonding between polyurethane and the CNT yarns used is investigated. To accomplish this a 4-ply yarn sample is bonded to polyurethane and encased in a separate adhesive and hardened. The sample is then polished to a high degree of smoothness and studied through a SEM. Representative SEM images are displayed in Figure 2 and 3 . The images show polyurethane penetration into the yarns around the perimeter of the yarn/polyurethane interface, but not deep within the yarn interior.

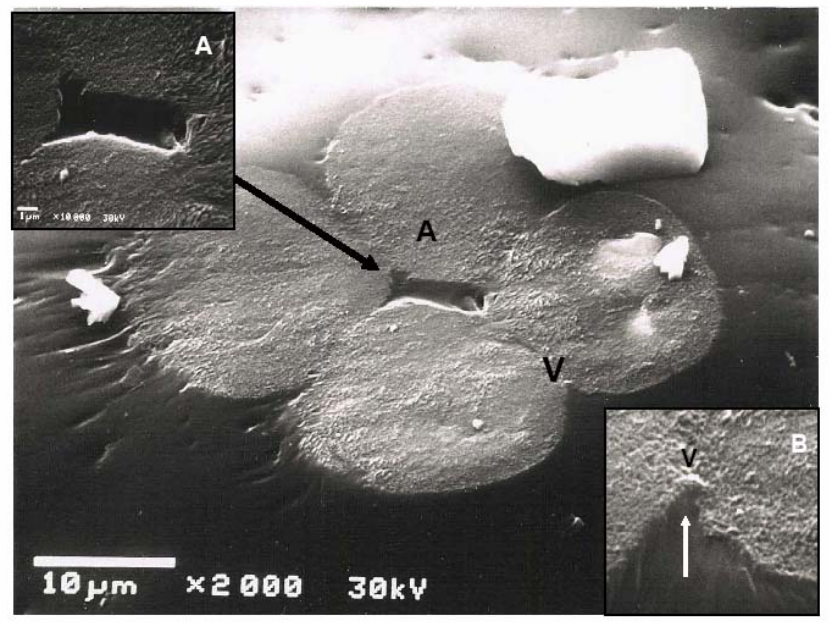

Figure 2. SEM images of a 4-ply yarn encased in polyurethane (REN 6405). The inset $A$ in the upper left indicates the polyurethane has not fully penetrated the yarn bundle, leaving the yarns free to deform internally. The inset $B$ in the lower right reveals polyurethane penetration along the outer walls preventing the yarn bundle from slipping under stain within the composite material.

In Figure 2, the inset A in the upper left corner shows the gap formed in the center of four single-ply yarns and indicates polyurethane did not fully penetrate yarn bundle and inset $\mathrm{B}$ shows the yarn looking along the direction arrow labeled "V." The inset B in the lower right corner indicates the polyurethane has penetrated the outer surface of the yarns bundle preventing the yarns from slipping within the composite while the sample is under strain.

Figure 3 shows the bonding between the 4-ply yarn and the polyurethane (ClearFlex 95) with the polyurethane removed from the yarn/polyurethane interface region during sample preparation, ClearFlex 95 has a Young's modulus of $17.24 \mathrm{MPa}$ and that of REN-6450 is 36.54 $\mathrm{MPa}$. Polyurethane penetration into the outer surface of the yarns has also been confirmed by removing the polyurethane by electron bombardment under SEM observation (B). Conversely, it is also confirmed that the polyurethane does not fully penetrate the 4-ply structure as illustrated by the air gaps in the center of the four yarns $(A)$.

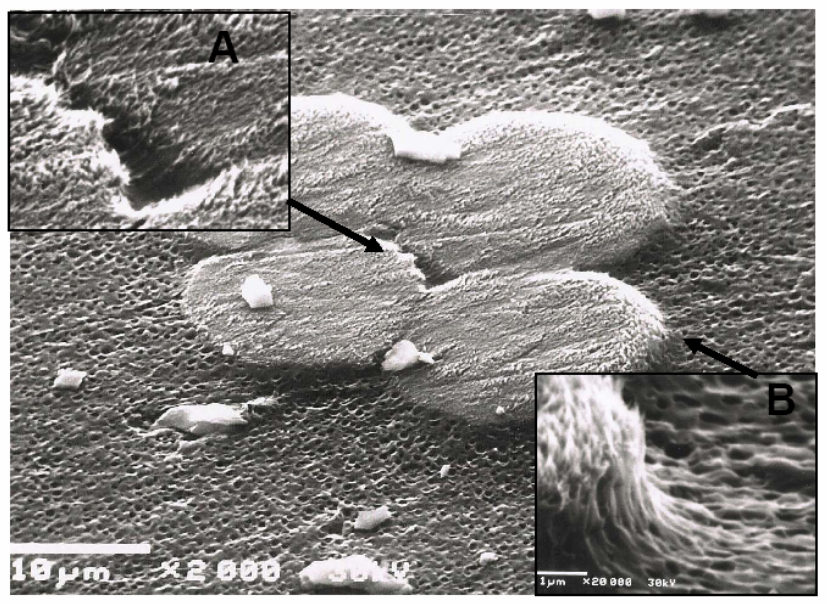

Figure 3. More SEM images of the 4-ply yarn cross section encased in polyurethane (ClearFlex 95).

An interesting physical property, from a sensing perspective, is the high Poisson's ratio observed by Baughman et al. ranging from 2.0 to 2.7 for multiwalled CNT singles yarn and from 3.3 to 4.2 for the two-ply yarn. Ordinary solids, including materials used in typical strain gages, have typical Poisson's ratios of $\sim 0.3$. With strain sensitivity directly related to two-times the Poisson's ratio, the potential use of yarns as embedded strain gages exists [8].

To evaluate the applicability of CNT yarns to strain sensing, CNT yarns are embedded on the surface of a stainless steel cantilever test beam utilizing polyurethane (REN 6405) as an adhesive. Typical test beams used in the test are $20.32 \mathrm{~cm}$ long, $2.54 \mathrm{~cm}$ wide and $0.318 \mathrm{~cm}$ thick stainless steel (17-4PH). The yarn is placed in a Wheatstone bridge electrical configuration while the cantilever is loaded in simple bending. The bridge output voltage is recorded and compared against standard metal foil strain gages placed on the test beam. Figure 4 shows a general description of the experimental setup.

The resultant output voltages, normalized by the output at 100 micro-strains, are shown in Figure 5 with input voltages varying from $0.3,0.5,0.7$, and 1.0 Volt. The results shown in Figure 5 show a linear correlation between the strain measured by the conventional strain gage and the voltage output of the bridge containing the CNT yarn. 


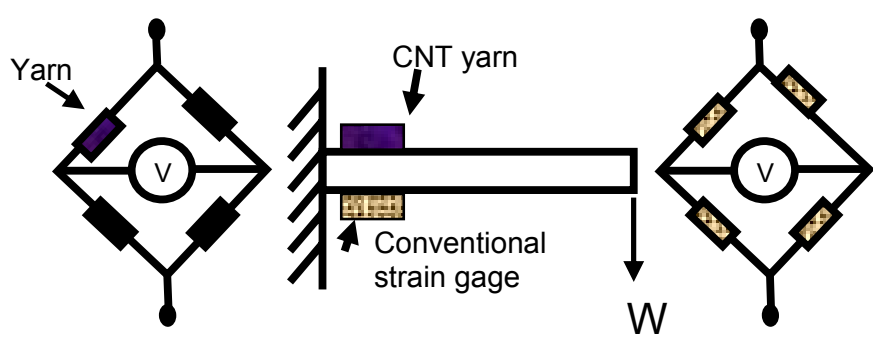

Figure 4. Diagrams of components outlining the experimental setup used to measure the response of the CNT yarns to applied strain on a cantilever test beam. The yarn is placed in a Wheatstone bridge and located on top of the cantilever test beam (left and center images). Four conventional strain gages are placed in a Wheatstone bridge configuration and placed on the bottom of the test beam (center and right images)

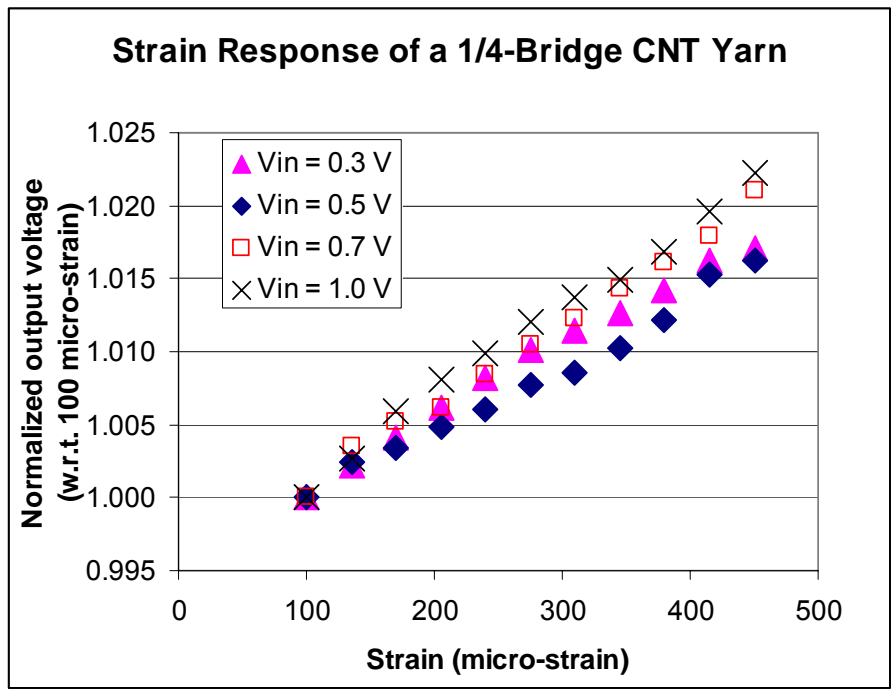

Figure 5. Response of Wheatstone bridge with CNT yarns to induced strain on a stainless steel cantilever test beam as measured by conventional strain gages. The bridge's output is normalized with respect to the output at 100 micro-strains.

Utilizing the data in Figure 5 to calculate a sensitivity (or gage factor) gives a value ranging from $1.65-2.75$. Table 1 compares that value to those for other types of strain gages.

Table 1: Comparison of strain gage sensitivities

\begin{tabular}{|c||c|c|}
\hline $\begin{array}{c}\text { Strain Gage } \\
\text { Type }\end{array}$ & Gage Factor & $\begin{array}{c}\text { Measurement Range } \\
\text { (Micro-strain) }\end{array}$ \\
\hline CNT Yarn & $1.65 \sim 2.75$ & $\sim 0.1 \sim 1,000$ \\
\hline Metal Foil & $2.0 \sim 4.5$ & $0.1 \sim 40,000$ \\
\hline Semiconductor & $50 \sim 200$ & $0.001 \sim 3,000$ \\
\hline
\end{tabular}

While a gage factor on the order of 2 is substantial, a larger sensitivity is both desirable and likely obtainable by eliminating problems. During the embedment process it was difficult to lay the yarns down exactly parallel to the measurement direction and without some degree of "waviness" along the length of the yarn. In addition, the degree of bonding between the yarns and polyurethane was poor, which could decrease stress transfer to the nanotube yarn. Both of these factors could lead to significant decrease of strain sensitivity.

The other aspect of the multifunctional characteristics of the described composites is increased mechanical strength due to yarn embedment. In order to evaluate the mechanical tensile strength CNT yarns are embedded into silicon rubber and polyurethane samples approximately 0.2 to $0.8 \mathrm{~mm}$ thick with gage lengths of $25.4 \mathrm{~mm}$. The samples are cut into a typical dog-bone geometry using a laser ablation method to ensure a uniform cut and a continuous edge as shown in Figure 6. The CNT yarns embedded in the polyurethane extends the entire length between both grip ends. The actual length and width of the test sample are $25.4 \mathrm{~mm}$ and $12.7 \mathrm{~mm}$, respectively.

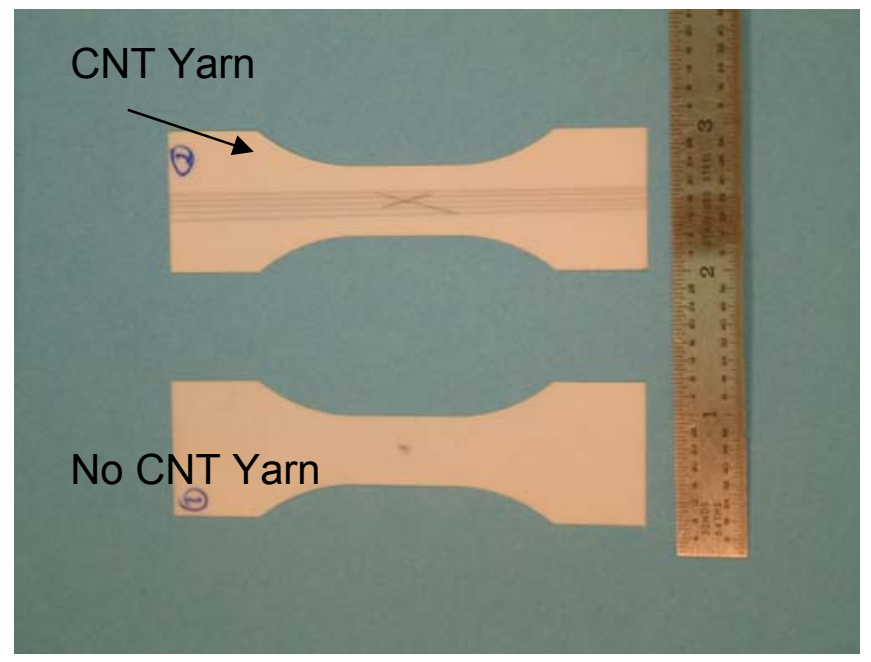

Figure 6. Test samples of thin polyurethane composites reinforced with the CNT yarns. The top sample has 5 CNT yarns embedded into the material and the bottom sample served as a reference and does not contain any yarns.

The samples are subjected to a uniaxial tensile test on an electro-mechanical test stand at room temperature using a constant applied displacement rate. Axial strain was measured with a non-contacting laser extensometer and load was measured with the test machine load cell. Stress was computed based on the average gage crosssection measured prior to test and strength was defined as a complete loss of load carrying capability during loading. Longitudinal elastic modulus was determined from the initial linear slope of the resultant stress-strain curve. An example of a typical result from this experiment is shown in Figure 7. Representative data of the experimentally determined tensile moduli are compared in Table 2. 


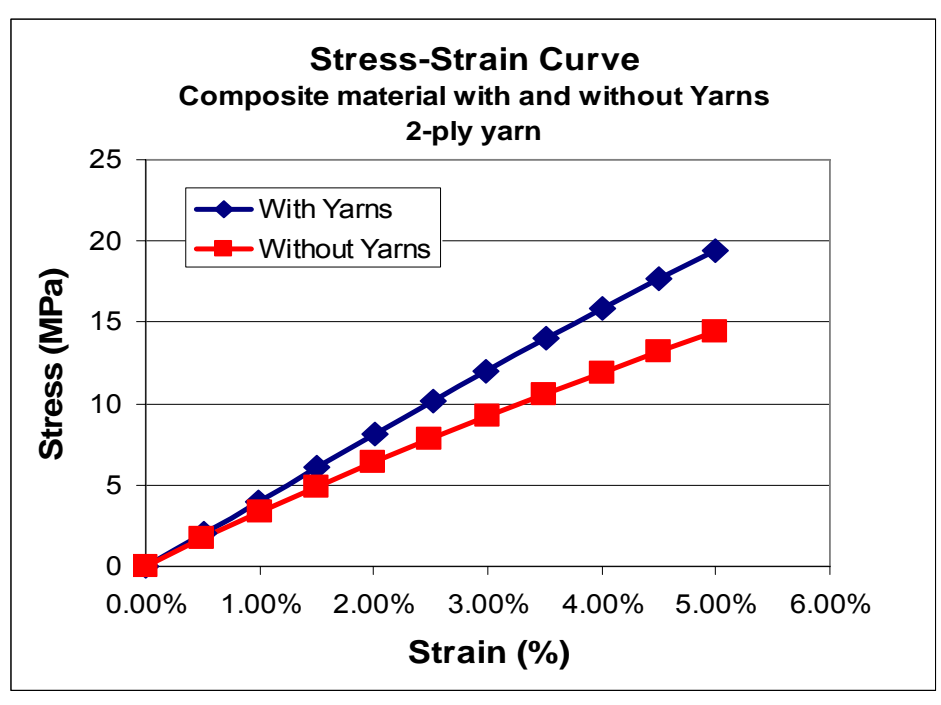

Figure 7. Typical stress-strain results of CNT yarn embedded/non-embedded test samples. Test results show an increase of longitudinal elastic modulus for the embedded over the non-embedded samples.

Table 2: Tabular results from representative mechanical strength tests comparing the moduli of CNT embedded polyurethane samples to the non-embedded test samples where $E_{n}$ is the material modulus without CNT yarns embedded, and $E_{y}$ is the material modulus with the embedded yarns representing the increase in modulus in comparison to the reference material.

\begin{tabular}{|c|c|c|c|c|c|c|}
\hline Samples & $\begin{array}{c}E_{n} \\
(\mathrm{MPa})\end{array}$ & $\begin{array}{c}\# \\
\text { Yarns }\end{array}$ & $\begin{array}{c}\mathrm{E}_{\mathrm{y}} \\
(\mathrm{MPa})\end{array}$ & $\begin{array}{c}\% \\
\text { weight } \\
\text { of } \\
\text { Yarn }\end{array}$ & $\begin{array}{c}\text { Mod. } \\
\text { Increase } \\
\text { Factor }\end{array}$ & $\begin{array}{c}\text { Thickness } \\
(\mathrm{mm})\end{array}$ \\
\hline $30-1$ & 11.94 & 14 & 13.99 & 0.023 & 1.17 & 0.8 \\
\hline $30-2$ & 4.02 & 6 & 1.51 & 0.020 & 0.38 & 0.8 \\
\hline $30-3$ & 1.16 & 6 & 2.89 & 0.013 & 2.49 & 0.6 \\
\hline $95-1$ & 2.46 & 10 & 1.79 & 0.070 & 0.73 & 0.19 \\
\hline $95-2$ & 2.46 & 20 & 2.29 & 0.132 & 0.93 & 0.20 \\
\hline $95-3$ & 4.02 & 22 & 6.1 & 0.037 & 1.52 & 0.8 \\
\hline $6405-1$ & 740 & 40 & 736 & 0.279 & 1.00 & 0.23 \\
\hline $6405-2$ & 740 & 20 & 737 & 0.095 & 1.00 & 0.26 \\
\hline $6405-3$ & 740 & 20 & 801 & 0.178 & 1.08 & 0.23 \\
\hline $6405-4$ & 672 & 20 & 921 & 0.098 & 1.37 & 0.25 \\
\hline $6405-5$ & 480 & 20 & 786 & 0.098 & 1.64 & 0.25 \\
\hline $6405-6$ & 198 & 10 & 345 & 0.070 & 1.74 & 0.18 \\
\hline
\end{tabular}

Preliminary results indicate that composites consisting of CNT yarns embedded into polyurethane have an increase in longitudinal tensile modulus of ranging from $0 \%-140 \%$ depending upon the material and weight percent of the CNT yarns when compared to baseline polyurethane data. Polyurethanes with three different modulus of elasticity are presented here as an illustration. In general the increase in modulus held for the first $10 \%$ of elongation, at that point the sample with and without yarn stress strain curves began to converge. While the data presented only represents a small data set, the data seems to indicate that for materials with moduli much smaller than the modulus of the yarn, the CNT yarns do enhance the mechanical strength of the composite. Berhan at el. estimated that expected increase in modulus with the yarn is close to $10 \%$ of the theoretical value due to waviness of the yarn [14]. Further investigation is required to better quantify this effect.

To further investigate the strain sensing properties of the yarns, a $16 \mathrm{~cm}$ by $16 \mathrm{~cm}$ silicon rubber membrane is fabricated with 5 evenly spaced CNT yarns embedded near the surface across the length of the membrane as illustrated in Figure 8. The square, flexible membrane is fixed on all sides with the center unsupported. A point deflection is applied to the center of the membrane while a constant current of $100 \mu \mathrm{A}$ is applied through the yarns. The center deflection is measured using a high accuracy laser displacement measuring device. The changes in yarn resistance versus center deflection are recorded.

The percent changes in resistance are then compared to the expected percentage of average strain determined using a finite element analysis on a finite element model of the membrane. The analysis is conducted using fixed edges with material properties similar to those of the silicon rubber used in the test. The values presented are derived from averaging nodal strains across the path of the yarn. The predicted strain values acquired by the analysis across the length of the yarn are shown figure 9. Comparative results of measured and predicted strain are shown in Figure 10. Figure 10 compares the percent change in resistance of the embedded yarns to the theoretically expected percent change in strain.

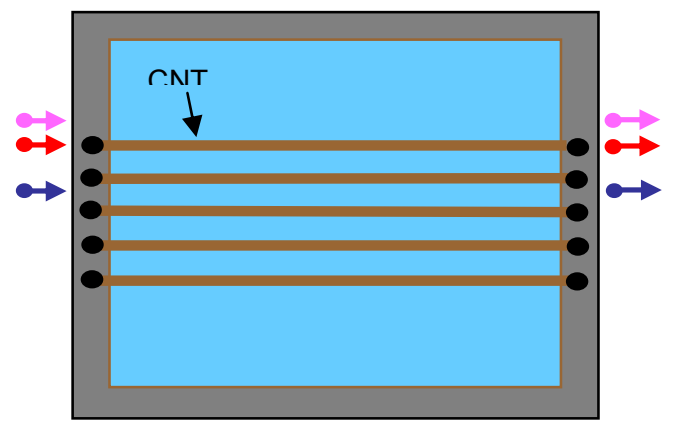

Figure 8. An illustration of the silicon rubber membrane used to investigate the strain sensing characteristics of the CNT yarns. 


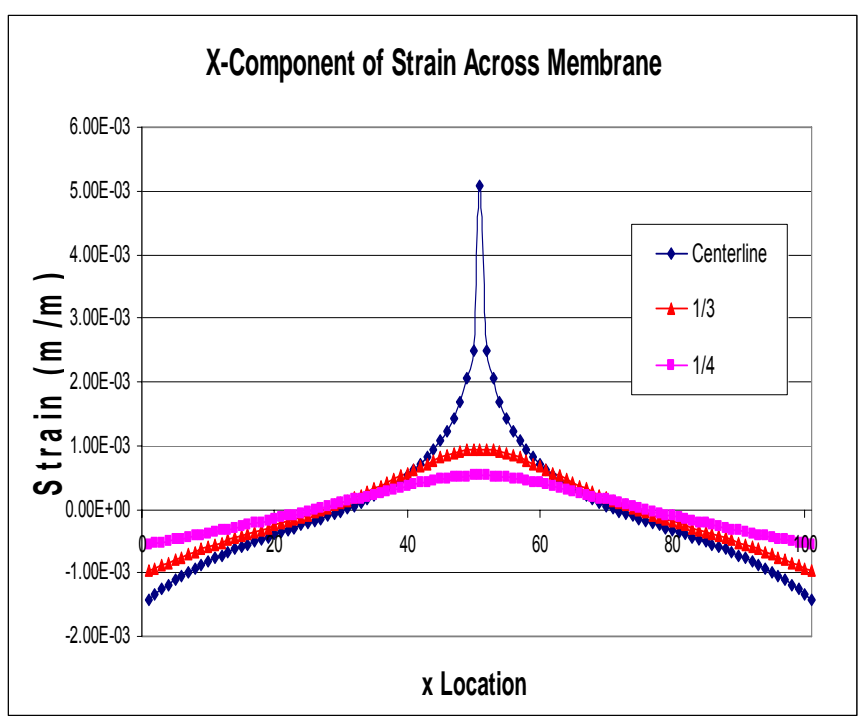

Figure 9. Predicted strain values across the length of the silicon membrane, at $y$ values of $50 \%, 30 \%$, and $25 \%$ of the membrane y-dimension, when the center is deflected 0.5 $\mathrm{mm}$.

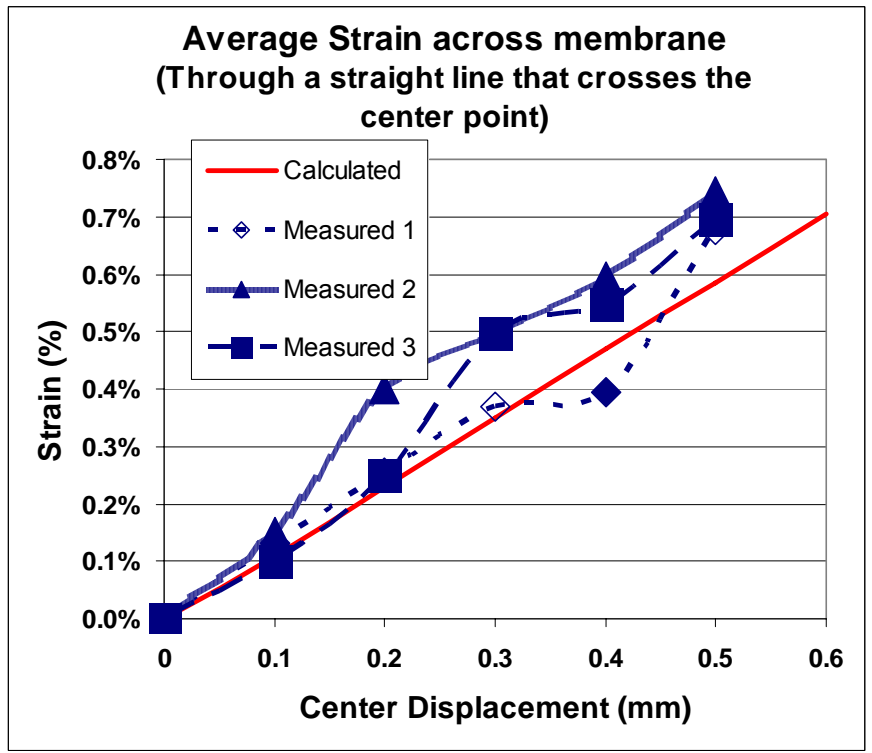

Figure 10. Comparison of predicted strain values in a thin, fixed membrane versus the measured percent change in resistance as a function of the displacement of the center of the membrane from a point force.

The sensitivity of nanotube yarn resistivity to temperature is low, which is advantageous for the strain sensor application [8].

For the temperature characterization experiment a CNT yarn is suspended between an electrode pair and exposed to ambient air in a thermal chamber. The chamber's temperature is varied between -60 degree $C$ and +130 degree $C$, and the CNT yarns are injected with currents of $1 \mu \mathrm{A}, 5 \mu \mathrm{A}, 100 \mu \mathrm{A}, 500 \mu \mathrm{A}$ and $1000 \mu \mathrm{A}$ at prescribed temperatures. Typical results of this experiment are illustrated in Figure 11 where we found the yarns to have a negative temperature dependence of resistance of approximately $0.07 \%$ per degree C for all but the lowest values of current. These results at high temperatures for low current and at all temperatures for high current are similar to previous results reported by Zhang et al. [8].

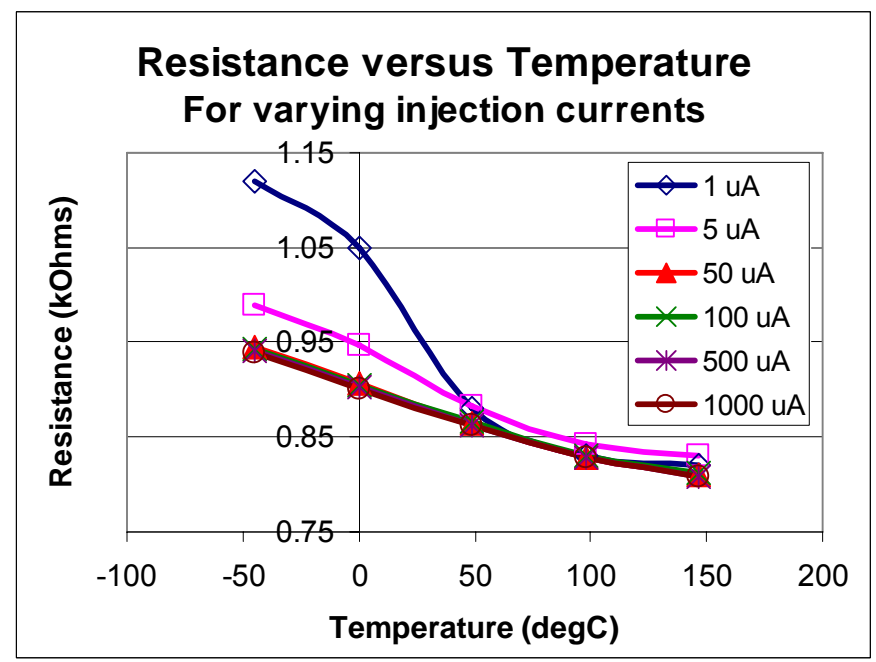

Figure 11. Resistance values of the yarns as a function of temperature for varying injection currents.

For the injection currents used, $(1 \mu \mathrm{A}, 5 \mu \mathrm{A}, 100 \mu \mathrm{A}, 500$ $\mu \mathrm{A}$, and $1000 \mu \mathrm{A})$, the estimated current densities range from 0.3184 to $159.2 \mathrm{Amp} / \mathrm{sq}-\mathrm{cm}$. The apparent resistances observed at low injection currents and low temperatures appear anomalous and differ from the more consistent values observed over the entire temperature range for much higher currents.

Discussed here are four experiments demonstrating the first steps towards utilizing carbon nanotube yarns as the basis of multifunctional composites. The results of the cantilever test beam and thin membrane experiment demonstrate the yarns ability to measure strain. Simultaneously, initial experiments show CNT yarns possess the ability to enhance the longitudinal elastic modulus of the investigated elastomers.

\section{References}

1. National Aeronautics and Space Administration 2005 Strategic Plan

2. R.H. Baughman, A.A. Zakhidov, W.A. de Heer, Science 297, 787 (2002)

3. P. Miaudet, S. Badaire, M. Maugey, A. Derre, V. Pichot, P. Launois, P. Poulin, C. Zakri, Nano Letters, 5, No. 11, 2212 (2005)

4. Y. Li, I. Kinloch, A. Windle, Science 2004. 304, 276

5. L. Ericson, H. Fan, H. Peng, V. Davis, W. Zhou, J. Sulpizio, Y. Wang, R. Booker, J. Vavro, C. Guthy, A. Parra-Vasquez, M. Kim, S. Ramesh, R. Saini, C. Kittrell, G. Lavion, H. Schmidt, W. Adams, W. 
Billups, M. Pasquali, W. Hwang, R. Hauge, J.

Fischer, R. Smalley, Science 305, 1447 (2000)

6. A. Dalton, S. Collins, E. Munoz, J. Razal, V. Ebron, J. Ferraris, J. Coleman, B. Kim, R. Baughman, Nature, 2003, 423, 703

7. B. Vigolo, A. Penicaud, C. Coulon, C. Sauder, R. Pailler, C. Journet, P. Bernier, P. Poulin, Science 290, 1331 (2000)

8. M. Zhang, K. Atkinson, R.H. Baughman, Science 306, 1358 (2004)

9. K. Jiang, Q. Li, S. Fan, Nature 419, 801 (2002)

10. Winters, J., Mechanical Engineering Magazine, 128 No. 4, 2006

11. R.H. Baughman, Science 308, 63 (2005)

12. Baughman, R.H., Science, 290, 1310, (2000)

13. M. Zhang, S. Fang, A. Zakhidov, S. Lee, A. Aliev, C. Williams, K. Atkinson, R. Baughman, Science 309, 1215 (2005)

14. Berhan, L. et al., J. of Appl. Phys, 95, 9, 5027 (2004) 